\title{
A Strategy to Assess the Cellular Activity of E3 Ligases against Neo-Substrates using Electrophilic Probes
}

Benika J. Pinch¹, Dennis L. Buckley¹, Scott Gleim¹, Scott M. Brittain¹, Laura Tandeske1, Pier Luca D'Alessandro ${ }^{1}$, Edward P. Harvey ${ }^{1}$, Zachary J. Hauseman ${ }^{1}$, Markus Schirle ${ }^{1}$, Elizabeth R. Sprague ${ }^{1}$, William C. Forrester ${ }^{1 *}$, Dustin Dovala ${ }^{1 *}$, Lynn M. McGregor ${ }^{1 *}$, Claudio R. Thoma ${ }^{1 *}$

${ }^{1}$ Novartis Institutes for BioMedical Research, Cambridge, MA 02139; Emeryville, CA 94608, USA.

*Correspondence: claudio.thoma@novartis.com (C.R.T.); lynn.mcgregor@novartis.com (L.M.M.); dustin.dovala@novartis.com (D.D.); william.forrester@novartis.com (W.C.F.) 


\begin{abstract}
Targeted protein degradation is a rapidly developing therapeutic modality that promises lower dosing and enhanced selectivity as compared to traditional occupancy-driven inhibitors, and the potential to modulate historically intractable targets. While the well-characterized E3 ligases CRBN and VHL have been successfully redirected to degrade numerous proteins, there are approximately 600 predicted additional E3 family members that may offer improved activity, substrate selectivity, and/or tissue distribution; however, characterizing the potential applications of these many ligases for targeted protein degradation has proven challenging. Here, we report the development of an approach to evaluate the ability of recombinant E3 ligase components to support neo-substrate degradation. Bypassing the need for hit finding to identify specific E3 ligase binders, this approach makes use of simple chemistry for Covalent Functionalization Followed by E3 Electroporation into live cells (COFFEE). We demonstrate this method by electroporating recombinant $\mathrm{VHL}$, covalently functionalized with JQ1 or dasatinib, to induce degradation of BRD4 or kinase targets, respectively. Furthermore, by applying COFFEE to SPSB2, a SOCS box and SPRYdomain E3 ligase that has not previously been redirected for targeted protein degradation, we validate this method as a powerful approach to define the activity of previously uncharacterized ubiquitin ligases against neo-substrates.
\end{abstract}




\section{INTRODUCTION}

Targeted protein degradation (TPD) involves co-opting the cell's endogenous protein turnover machinery by redirecting a ubiquitin E3 ligase to engage a protein of interest (POI) and promote its subsequent proteolysis at the proteasome. This emerging therapeutic modality promises to expand the landscape of druggable targets by enabling the selective removal of proteins that have been historically difficult to modulate with small molecules, such as proteins with scaffolding functions, or those in closely related families. For example, degradation of the BAF complex component, BRD9, revealed an essential scaffolding role in leukemia and synovial sarcoma that was not observed with small molecule bromodomain inhibitors (Brien et al., 2018; Remillard et al., 2017); and a dual CDK4/6 inhibitor was transformed into selective CDK4 or CDK6 degraders by linking the non-selective inhibitor, palbociclib, to the cereblon (CRBN)-recruiting ligand, thalidomide (Jiang et al., 2019). Furthermore, degraders offer sub-stoichiometric catalytic activity, enabling efficacy at lower doses than their parental inhibitors (Li et al., 2020).

While there are approximately 600 ubiquitin E3 ligases spanning many structural families, ligands for just two substrate receptors, CRBN and von Hippel-Lindau (VHL), have driven rapid expansion of this novel pharmacology, while also limiting its broader applications to other E3s. (Schapira et. al. 2019). Recent work highlights the untapped potential of the E3 family for TPD, with reports that previously unexplored E3 ligase components can be hijacked to induce neo-substrate degradation, such as RNF114 (Spradlin et al., 2019), DCAF16 (Zhang et al., 2019), RNF4 (Ward et al., 2019), and DDB1 (Slabicki et al., 2020). Expanding the TPD toolkit to include ligands for additional E3 ligases could enable tissue- and disease-specific degradation according to E3 ligase expression patterns (Schapira et al., 2019), could broaden the landscape of degradable targets by presenting distinct E3 topologies for ternary complex formation, and/or could offer E3 ligands with improved physicochemical properties.

Structural studies and molecular dynamics simulations have provided insight regarding the dynamics of Cullin-RING E3 ligase-mediated ubiquitination of substrates docked at the receptor. Movement of the flexible RBX/ubiquitin E2 arm and E3 substrate binding domain facilitates scanning of the substrate's surface accessible lysines for ubiquitin addition, and suggests that the geometry of the substrate-E3 interaction is a critical determinant of efficient ubiquitin transfer (Liu et al., 2009). To our knowledge, there are no reported assays that test the suitability of specific sites on E3 ligases for pharmacological redirection to degrade neo-substrates in living cells. Evaluating the utility of an E3 ligase for TPD requires either genetic engineering of the E3 ligase of interest (Ottis et al., 2017), or the discovery of an E3 ligand followed by the incorporation of that ligand into a bifunctional degrader through additional rounds of medicinal chemistry. We therefore sought to develop a TPD assay to test the "hijackability" of an E3 ligase prior to resource-intensive genetic manipulation or hit-finding chemistry campaigns.

Over 400 E3 ligases have at least one cysteine, making them potentially amenable to covalent targeting. Accordingly, several covalent E3 ligase ligands have been reported and successfully incorporated into bifunctional degraders (Ward et al., 2019; Spradlin et al., 2019; Zhang et al., 2019). While identifying covalent ligands for E3 ligases has proven relatively straightforward, non-optimized covalent ligands often lack selectivity, resulting in off-target activity. While this can be overcome by selective covalent labeling of the purified, recombinant E3, the resulting functionalized E3 is not expected to be cell permeable. In order to develop a probe that would engage various structurally unrelated E3 ligases, and that would enable selective cellular readouts, we sought to append recombinant E3 ubiquitin ligases with covalent probes, and then electroporate the labeled proteins into cells for TPD exploratory studies. Previous reports showcase the utility of electroporation as a relatively gentle method for introducing nucleotides (Tsong et al., 1989), 
peptides (Schönenberger et al., 2011), proteins (Alex et al., 2019), antibodies (Clift et al., 2018), and various other cell-impenetrant substances into live cells.

In this work, we demonstrate that E3 ligases, including VHL and SPSB2, can be functionalized via their solvent-exposed cysteines using a simple maleimide warhead linked to either the BRD4 ligand, JQ1, or the multi-kinase inhibitor, dasatinib. We further show that the resulting functionalized recombinant E3 ligases can then be electroporated into live cells to form functional E3 ubiquitin ligase complexes capable of catalyzing POI degradation. This method, Covalent Functionalization Followed by E3 Electroporation (COFFEE), provides proof-of-concept information about ligase hijackability that can facilitate the prioritization of E3 ligases for TPD studies and ligandfinding campaigns.

\section{RESULTS}

\section{Electroporation of Recombinant E3 Ligases Yields Functional CRL Complexes}

To identify E3 ligases potentially amenable to COFFEE, we used a bioinformatics approach to identify all E3 ligase components containing cysteines. We then filtered out E3 ligases that catalyze direct transfer of ubiquitin from an E2 to the target protein via an active site cysteine, such as HECT E3s, since modification of the reactive cysteine would presumably inhibit E3 ligase activity (Chen et al., 2018). Finally, we prioritized E3 ligases based both on the availability of structural data, and on a low total cysteine count in order to minimize multiple labeling events (Fig 1A). Using this approach, VHL emerged as a top candidate for functionalization with neo-substrate ligands, having only two cysteines and a well-characterized three-dimensional structure (Hon et al., 2002). Furthermore, as a validated E3 ligase for TPD applications (Bondeson et al., 2015; Zengerle et al., 2015; Gechijian et al., 2018; Bond et al., 2020), VHL presented an ideal proof-of-concept E3 ligase for COFFEE. We therefore expressed purified recombinant VHL in complex with the Cullin-RING ligase $(C R L)$ adaptor proteins, Elongin $B(E l o B)$ and Elongin $C(E l o C)$, which are required for protein stability. While native VHL exists as two isoforms due to an alternate translation initiation site, we produced the highly expressed and biologically active short isoform (lliopoulos et al., 1998). To avoid functionalization of the solvent exposed Cys89 of EloB, we also expressed and purified a serinesubstituted version, VHL/EloB ${ }^{\mathrm{C} 89 \mathrm{~S}} /$ EloC.

While prior reports demonstrate that electroporated recombinant proteins are functional and exhibit native localization (Alex et al., 2019), to our knowledge, it has not been previously demonstrated that electroporated E3 ligases can associate with endogenous components to form functional ubiquitination complexes. Therefore, following optimization of the electroporation conditions using fluorescently labeled BSA (Fig. S1), we sought to assess whether electroporated $\mathrm{VHL} / \mathrm{EloB}^{\mathrm{C} 89 \mathrm{~S}} / \mathrm{EloC}$ engages Cullin 2 (Cul2)-Rbx1 to form the multimeric CRL2 ${ }^{\mathrm{VHL}}$ complex. To this end, we performed a co-immunoprecipitation (co-IP) following electroporation of biotin$\mathrm{VHL} / \mathrm{EloB}^{\mathrm{C} 89 \mathrm{~S}} / \mathrm{EloC}$ (Fig. S1) into the renal cell carcinoma 786-O cell line, which harbors a $\mathrm{VHL}$ mutation and therefore does not express VHL protein (lliopoulos et al., 1995). Biotin$\mathrm{VHL} / \mathrm{EloB}^{\mathrm{C} 89 \mathrm{~S}} / \mathrm{EloC}$ pulled down Cul2 and Rbx1, thereby demonstrating successful assembly of the full CRL2 $2^{\mathrm{VHL}}$ complex following electroporation. By contrast, electroporation of negative controls, including buffer alone or non-biotinylated $\mathrm{VHL} / \mathrm{EloB}^{\mathrm{C} 89 \mathrm{~S}} / \mathrm{EloC}$, did not pull-down Cul2 or Rbx1 (Fig. 1B).

We next evaluated whether electroporated $\mathrm{VHL} / \mathrm{EloB}^{\mathrm{C} 89 \mathrm{~S}} / \mathrm{EloC}$ resulted in a functional $\mathrm{CRL}$ complex, capable of inducing substrate degradation. As compared to non-electroporated cells or cells electroporated with recombinant BSA as a negative control, electroporation of $\mathrm{VHL} / \mathrm{EloB}^{\mathrm{C} 89 \mathrm{~S}} / \mathrm{EloC}$ into $786-\mathrm{O}$ cells decreased levels of VHL's canonical substrate, HIF2 $\alpha$ (Tarade et al., 2019), matching the reduced HIF2 $\alpha$ expression observed in 786-O cells expressing both 
isoforms of VHL from a lentiviral vector (Fig. 1C). Electroporation of WT VHL/EloB/EloC similarly reduced HIF2 $\alpha$ expression in $786-\mathrm{O}$ cells (Fig. S2), highlighting that the EloB ${ }^{\mathrm{C} 895}$ mutant does not affect functionality of the CRL complex. Successful electroporation of VHL into cells was monitored by immunoblot using an anti-VHL antibody. Taken together, these data confirm that recombinant E3 ligases can be electroporated into cells to form functional E3 ligase complexes capable of mediating ubiquitination and protein degradation through the canonical proteasome-dependent pathway.

\section{Electroporation of JQ1-functionalized VHL induces BRD4 degradation}

Having confirmed that recombinant, electroporated $\mathrm{VHL}$ degrades its native substrate, we next tested whether we could use simple cysteine-maleimide chemistry to extend this approach to neo-substrate degradation (Fig. 1D). VHL contains two cysteines, with Cys 77 adjacent to the native substrate binding site, and Cys162 buried at the interface between VHL and EloC (Fig. 2A) (Hon et al., 2002). Given that only Cys 77 is solvent exposed, VHL should be suitable for facile single labeling with electrophiles, presenting an opportunity to chemically modify the surface of VHL for TPD studies.

To functionalize VHL Cys77, we synthesized Compound 1, a bifunctional compound comprised of a cysteine-reactive maleimide connected to the BRD4 ligand, JQ1, via a 2-PEG linker (Fig. 2B) (Filippakopoulous et. al., 2010). Selective and complete single labeling of VHL by 1 was confirmed by intact protein mass spectrometry (Fig. 2C). Electroporation of the resulting VHL1/EloB ${ }^{\mathrm{C} 89 S} /$ EloC complex into HEK293A cells led to the dose-dependent loss of BRD4, as assessed by immunoblot analysis (Fig. 2D). Notably, VHL-1 showed an upward shift in the VHL band by immunoblot as compared to native recombinant VHL, consistent with its increased molecular weight. A panel of negative controls did not affect BRD4 protein levels, including electroporation of buffer alone, unmodified VHL/EloB ${ }^{\mathrm{C} 89 S} / \mathrm{EloC}$, or BSA-1 (Fig. 2D; Fig. S2). Furthermore, incubation of cells with $\mathrm{VHL}-1 / \mathrm{EloB}^{\mathrm{C} 89 \mathrm{~S}} / \mathrm{EloC}$ without electroporation did not affect BRD4 levels, highlighting that electroporation is necessary for cellular uptake (Fig. S2).

Pretreatment with either the proteasome inhibitor, carfilzomib, or the NEDD8-activating enzyme 1 (NAE1) inhibitor, MLN4924, rescued VHL-1/EloB ${ }^{\mathrm{C} 89 S} /$ EloC-mediated BRD4 degradation, reflecting a dependence on active $C R L$ activity and proteasomal degradation. Furthermore, pretreatment with an excess of JQ1 $(10 \mu \mathrm{M})$ also rescued BRD4 degradation, confirming a requirement for BRD4 engagement (Fig. 2F). To evaluate the kinetics of degradation, we performed a time course in HEK293A cells, in which cells were collected 6, 24, 48, or 72 hrs postelectroporation of $\mathrm{VHL}-1 / \mathrm{EloB}^{\mathrm{C} 89 \mathrm{~S}} / \mathrm{EloC}$ (Fig. 2E). The quantity of electroporated VHL-1 declined quickly, and was undetectable by $72 \mathrm{~h}$, in accordance with VHL's reported half-life (Zecha et al., 2018; Pozzebon et al., 2013). As expected, the levels of BRD4 recovered as the levels of electroporated VHL-1 decreased. This highlights that COFFEE must be applied within the half-life of the electroporated protein in order to detect neo-substrate degradation. Taken together, these results confirm that electroporated recombinant $\mathrm{VHL}-1$ induces the proteasomal degradation of BRD4.

\section{Electroporation of Dasatinib-functionalized VHL induces degradation of kinase targets}

Substrate ubiquitination by cognate E3 ligases or via bifunctional degraders requires the formation of transient complexes. Accordingly, the successful ubiquitination of neo-substrates often reflects their ability to form a de novo protein-protein interface with the recruited E3 ligase (Gadd et al., 2017; Nowak et al., 2018). For example, recent studies show that degradation by bifunctional multi-kinase degraders is dependent on stable ternary complex formation, and that degradation selectivity varies according to the recruited E3 ligase (Tong et al., 2020; Bondeson et al., 2018; Lai et al., 2016). We were therefore interested in using our maleimide-conjugation strategy to extend 
COFFEE to additional neo-substrate targets by appending VHL with dasatinib, a promiscuous kinase inhibitor that engages 38 kinases with an apparent $\mathrm{Ki}<100 \mathrm{nM}$, in addition to its primary target, BCR-ABL (Klaeger et al., 2017; Smith, et. al., 2019).

In order to extend our approach to additional neo-substrates, we synthesized a maleimidelinked dasatinib probe, Compound 2, using the reported exit vector for dasatinib-based bifunctional degraders (Tong et al., 2020). Compound $\mathbf{2}$ gave complete single labeling of $\mathrm{VHL} / \mathrm{EloB}^{\mathrm{C} 89 \mathrm{~S} / E l o C}$, as monitored by intact mass spectrometry (Fig. 3A, 3B). We next evaluated the effects of electroporated VHL-2/EloB ${ }^{\mathrm{C} 89 \mathrm{~S}} / \mathrm{EloC}_{\mathrm{O}}$ on ABL1 and Lyn kinase, which are among dasatinib's top 10 high-affinity targets (Montenegro et al., 2020; Klaeger et al., 2017; Leonard et al., 2016).

Electroporation of $\mathrm{VHL}-2 / \mathrm{EloB}^{\mathrm{C} 89 S} / \mathrm{EloC}$ induced the concentration-dependent loss of both ABL1 and

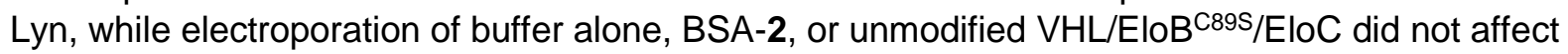

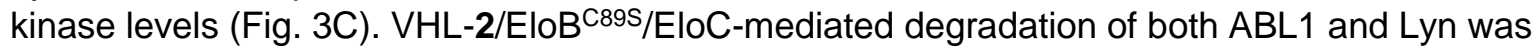
rescued upon pretreatment with carfilzomib or NAE1i, confirming that degradation occurs through the expected mechanism (Fig. 3D). Degradation of $A B L 1$ was also rescued upon pretreatment with an excess of dasatinib $(1 \mu \mathrm{M})$, validating a dependence on $A B L 1$ target engagement. Dasatinib treatment alone led to loss of Lyn kinase, likely due to transcriptional effects from broad kinase inhibition at this concentration, or ligand induced protein destabilization. These data highlight that COFFEE can be extended to include ligands against diverse neo-substrates, and can be used to further evaluate the landscape of degradable kinases.

\section{SPSB2 is a previously uncharacterized E3 ligase for TPD applications}

While VHL provided an ideal proof-of-concept E3 ligase for COFFEE, we next asked whether COFFEE could be applied to a different E3. We therefore expressed and purified another SOCS box E3 ligase containing surface-exposed cysteines, SPSB2, in complex with EloBC (SPSB2/EloB/EloC). SPSB2 is a validated E3 ligase and negative regulator of inducible nitric oxide synthase (iNOS), but has not previously been evaluated for activity against neo-substrates (Kuang et al., 2010). While SPSB2 contains four cysteines, only three are solvent exposed (PDB 5XN3) (You et al., 2017). Whereas stoichiometric reaction with 2 led to multiple labeling events, we established reaction conditions using sub-stoichiometric 2 that led to $\sim 50 \%$ single labeling of SPSB2 Cys53, as monitored by intact protein mass spectrometry (Figs. 4A, 4B, S3). Electroporation of the resulting SPSB2-2/EloB/EloC complex into HEK293A cells led to the dose-dependent decrease of ABL1 and Lyn kinases, while electroporation of buffer, BSA-2, or unmodified SPSB2/EloB/EloC did not alter kinase levels (Fig. 4C). As expected, SPSB2-2/EloB/EloC-mediated kinase degradation was rescued upon pretreatment with carfilzomib or NAE1i (Fig. 4D).

Next, we performed a quantitative proteomics experiment to ask whether other targets of dasatinib might also be degraded by SPSB2-2/EloB/EloC. For this experiment, we also selectively modified EloB Cys89 with 2 in order to evaluate the ability of this adaptor component to direct neosubstrate degradation. To do so, we expressed a complex of $\mathrm{VHL}^{\mathrm{C} 77 \mathrm{~S} / E l o B / E l o C}$, in which EloB Cys89 is the only reactive cysteine (Fig. S3). HEK293A cells were electroporated with SPSB22/EloB/EloC, VHLC77S/EloB-2/EloC, or BSA-2 and incubated for six hours. The resulting lysates were isotopically labeled with TMT reagents and analyzed by nano-LC/MS/MS. The protein abundance ratios (log2) were calculated for SPSB2-2 vs. BSA-2 and for EloB-2 vs. BSA-2. The comparison of these ratios demonstrates that electroporation of SPSB2-2 led to degradation of EPHB2, EPHB4, $A B L 1$, and $A B L 2$, all known targets of dasatinib, as well as moderate degradation of Lyn kinase, in agreement with the immunoblot results (Fig. 4D). Electroporation of $\mathrm{VHL} \mathrm{C}^{77 S / E l o B-2 / E l o C}$ also led to a modest decrease in the abundance of EPHB2 and EPHB4, highlighting the potential for this E3 adaptor protein to directly mediate neo-substrate degradation. Many other dasatinib targets (Klaeger 
et al., 2017) were identified in this experiment but were not degraded by SPSB2-2/EloB/EloC or by VHLC77S/EloB-2/EloC.

\section{DISCUSSION}

Recent advances in human genetics research using genome-wide CRISPR loss-of-function or RNAi screens have provided insight into protein dependencies across various diseases (McDonald et. al., 2017; So et al., 2019). Pharmacological efforts to modulate targets by a similar loss-of-function approach have fueled attempts to adapt targeted protein degradation as complementary to classic orthosteric inhibition. However, the field of targeted protein degradation must gain a better understanding of the rules that govern productive combinations of E3 ligase components, target proteins, and chemical matter.

The validation of E3 ligases beyond CRBN and VHL opens the door to novel E3 ligand discovery, which could provide an avenue for optimizing bifunctional degraders beyond the standard "linkerology", and could expand the landscape of degradable targets. Furthermore, while VHL and CRBN are ubiquitously expressed, recruiting E3 ligases with tissue- or disease-specific expression could improve degradation selectivity and minimize compound-associated toxicity. Therefore, a method to evaluate the ability of functionalized recombinant E3 ligases to catalyze degradation of neo-substrates of interest would enable rapid prioritization of E3s for follow-up ligand finding campaigns. Here, we report the development of such a method, termed Covalent Functionalization Followed by E3 Electroporation (COFFEE), to assess the ability of covalently functionalized recombinant E3 ligases to promote neo-substrate degradation. As proof-of-concept, we demonstrated that electroporated VHL-JQ1 or VHL-dasatinib conjugates successfully induced the proteasome-dependent degradation of BRD4 and kinase targets, respectively. We further extended this approach to validate neo-substrate degradation by SPSB2, demonstrating that this E3 ligase can also be hijacked for TPD applications. There are existing crystal structures of SPSB2 bound by cyclic peptide inhibitors, highlighting the chemical tractability of this E3 (You et al., 2017). Furthermore, the SPSB2 cysteine labeled using COFFEE, Cys53, is unique amongst the SPSB family members (Fig. S3), making the development of selective, covalent SPSB2 ligands another possible path to ligand discovery. Finally, we also demonstrated modest neo-substrate degradation by an EloB-dasatinib conjugate, highlighting that this E3 adaptor component can catalyze degradation, potentially bypassing the need for a substrate receptor. Although we studied just three E3 ligase components, COFFEE relies on simple chemistry, which can be applied to any recombinant E3 ligase component that contains reactive cysteines. Furthermore, the development of a broader set of maleimide-linked ligands, of varying linker lengths and polarities, would further extend the capabilities of COFFEE given that neo-substrate degradation is often target- and linkerdependent (Li et al., 2020).

It is likely that many E3 ligase components possess multiple reactive cysteines, and therefore site-specific modification of such ligases may require the generation of "cysteine-scrubbed" mutant proteins. Such a series of mono-cysteine constructs could enable researchers to evaluate the ability of multiple faces of an E3 ligase component to support targeted protein degradation, as well as the orientation-specific selectivity of degradation. We believe the question of optimal orientation of neo-substrate recruitment can be uniquely addressed by COFFEE, as related methods relying on engineered protein complexes do not offer a similar level of resolution. Furthermore, it is conceivable that preliminary exploratory studies using COFFEE could accelerate the subsequent path to bifunctional degrader development by directly validating particular E3 cysteines for the generation of corresponding covalent ligands or, more generally, by pinpointing E3 binding pockets suitable for ligand finding campaigns. In addition, the composition of the maleimide-linked probes used for COFFEE could inform optimal bifunctional degrader linker length to accelerate medicinal 
chemistry optimization efforts. We envision that future applications of COFFEE to diverse E3 ligase components will facilitate the expansion of the TPD toolkit beyond ligands for the widely characterized CRBN and VHL, and will accelerate our understanding of the requirements for efficient and selective neo-substrate degradation.

\section{MAIN TEXT FIGURES}

\section{Figure 1}

A

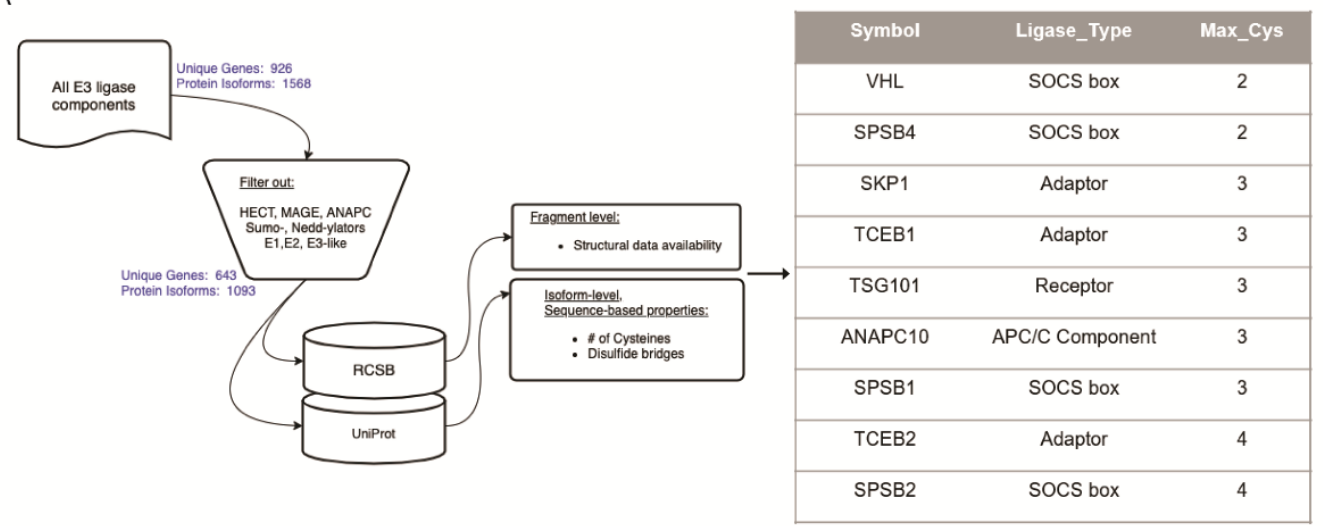

B

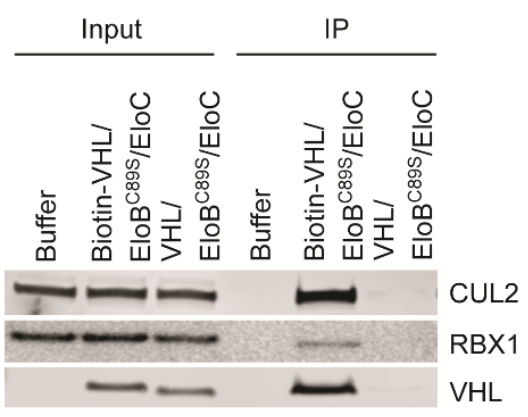

C

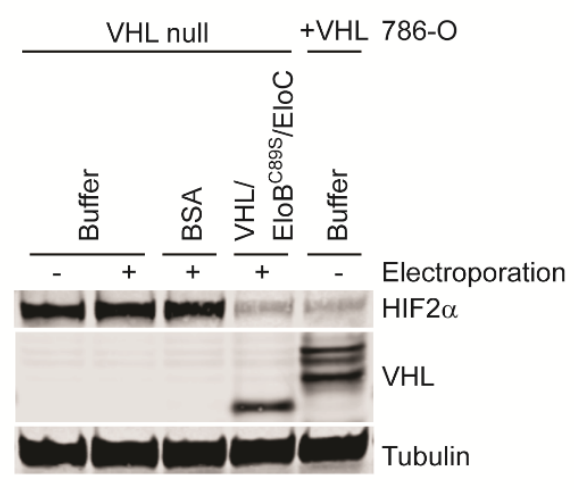

D

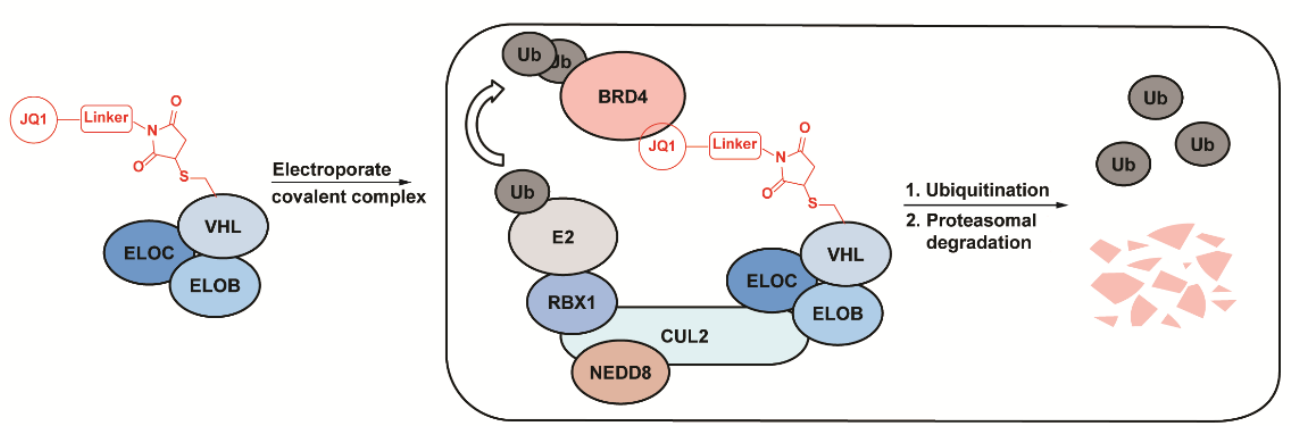




\section{Figure 2}

A

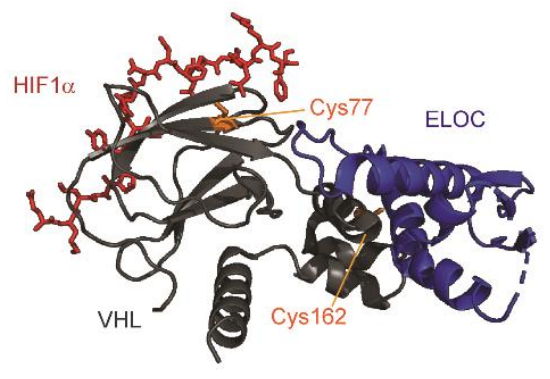

D

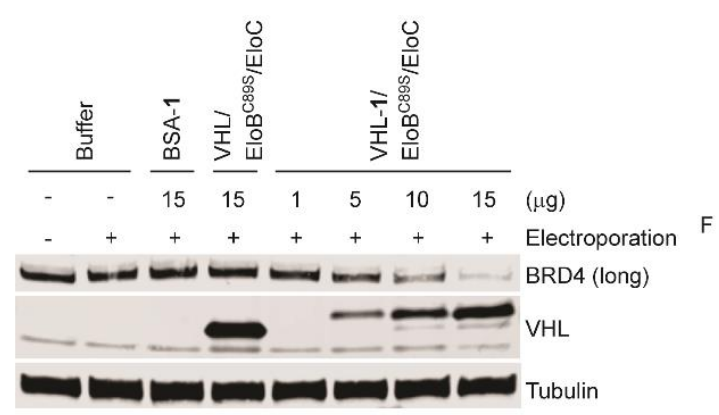

C

E
B
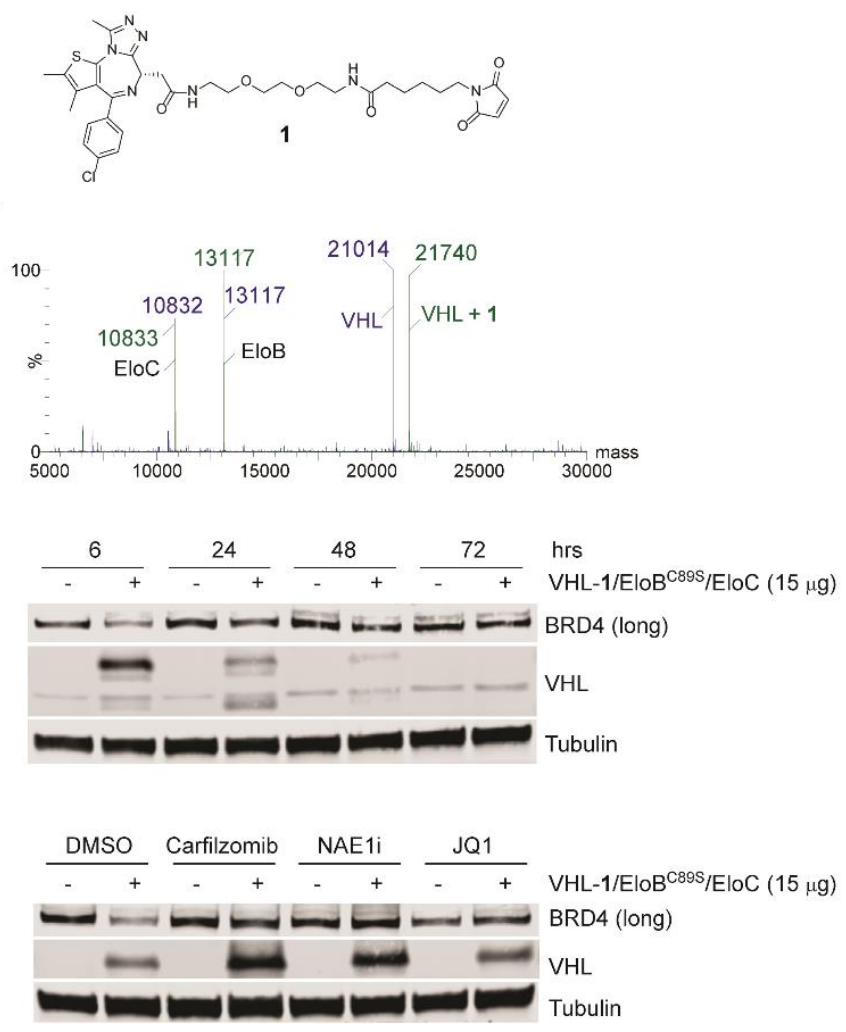
bioRxiv preprint doi: https://doi.org/10.1101/2020.08.13.249482; this version posted August 14,2020 . The copyright holder for this preprint (which was not certified by peer review) is the author/funder. All rights reserved. No reuse allowed without permission.

\section{Figure 3}

A

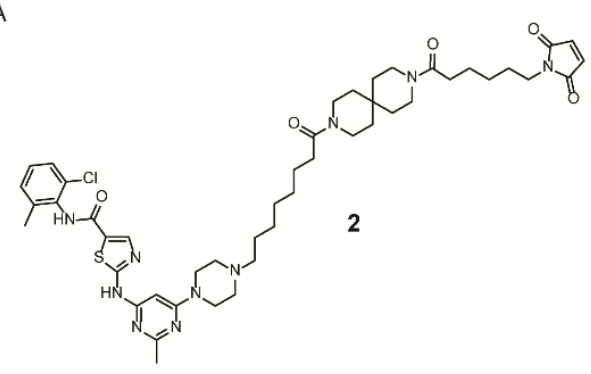

C

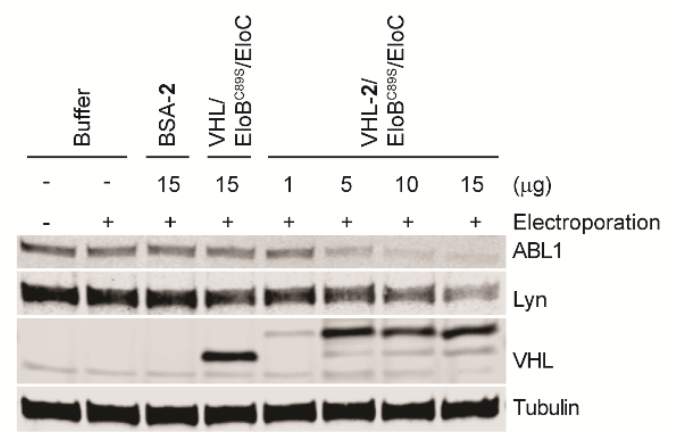

B

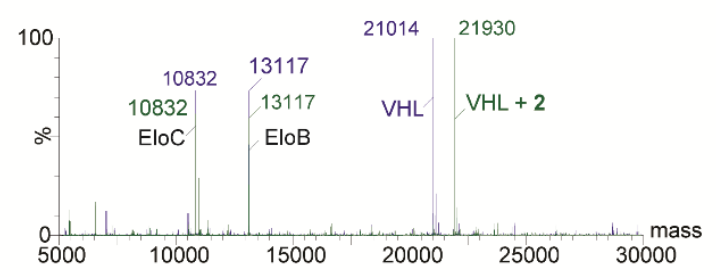

D

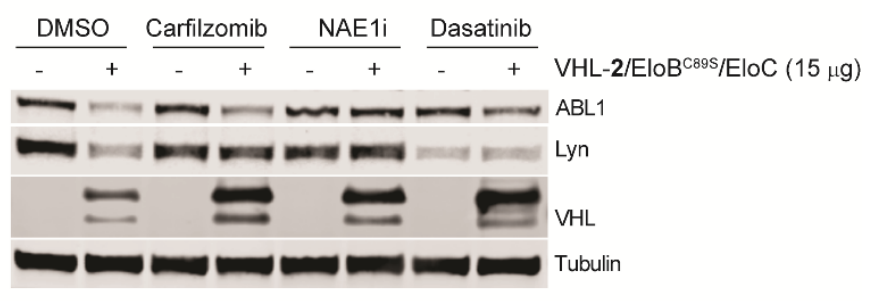




\section{Figure 4}

A

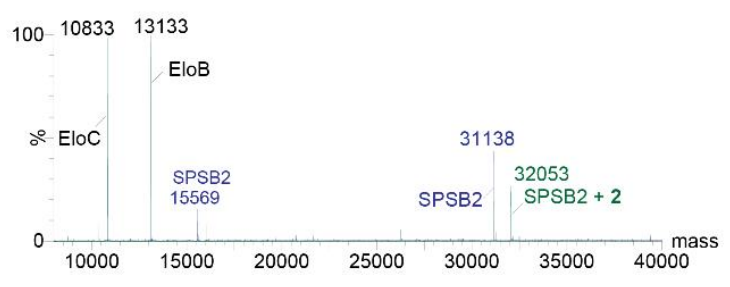

C

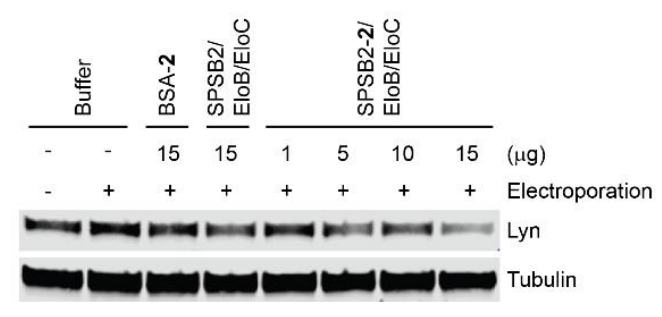

D

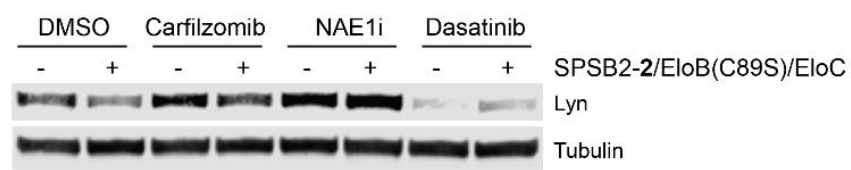

B

E
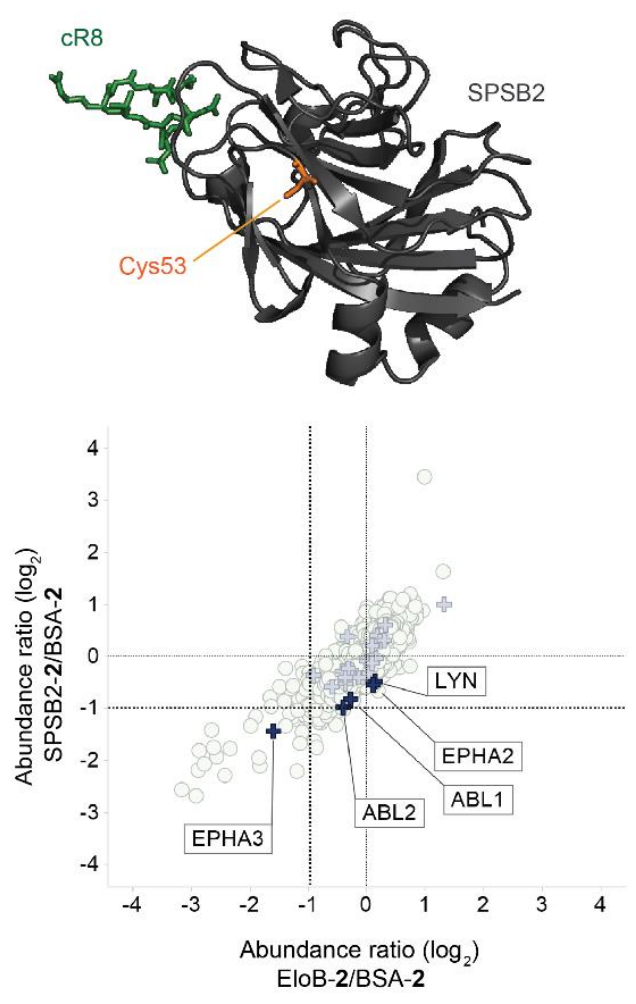


\section{FIGURE LEGENDS}

\section{Figure 1. Electroporated VHL is Functional.}

A. E3 ligase components with potentially ligandable cysteines were identified using a bioinformatics approach.

B. Co-IP with streptavidin agarose resin in 786-O cells following electroporation of buffer, biotin$\mathrm{VHL} /$ EloB $^{\mathrm{C} 89 \mathrm{~S}} /$ EloC, or VHL/EloB ${ }^{\mathrm{C} 89 \mathrm{~S} / E l o C .}$

C. Immunoblot analysis following electroporation of buffer, BSA, or VHL/EloB ${ }^{\mathrm{C} 895} /$ EloC into 786O cells, as compared to basal HIF2 $\alpha$ expression levels in 786-O cells transduced with VHL.

D. Schematic depiction of COFFEE workflow, as applied to VHL.

Figure 2. Degradation of BRD4 by JQ1-Functionalized VHL

A. Of the two VHL cysteines (highlighted in orange), Cys77 is adjacent to the native substrate binding site and Cys162 is buried in the interface between VHL and EloC. VHL is shown in gray and Elongin C (ELOC) in blue. Image from PDB 1LQB (Hon et al., 2002).

B. Chemical structure of Compound 1, a maleimide-JQ1 probe.

C. Intact mass spectrometry showing complete and specific labeling of VHL by 1. Unconjugated $\mathrm{VHL} / \mathrm{EloB}^{\mathrm{C} 89 \mathrm{~S}} / \mathrm{EloC}$ is shown in blue, and $\mathrm{VHL}-1 / \mathrm{EloB}^{\mathrm{C} 89 \mathrm{~S}} / \mathrm{EloC}$ in green.

D. Immunoblot analysis showing electroporation of $\mathrm{VHL}-1 / \mathrm{EloB}^{\mathrm{C} 89 \mathrm{~S}} / \mathrm{EloC}$ into HEK293A cells led to a dose-dependent decrease in BRD4, as compared to cells electroporated with buffer, BSA1, or unlabeled $\mathrm{VHL} / \mathrm{EloB}^{\mathrm{C} 89 \mathrm{~S}} / \mathrm{EloC}$. Doses are represented as $\mu \mathrm{g}$ of recombinant protein electroporated per well.

E. Immunoblot analysis of HEK293A cells pretreated with DMSO, carfilzomib (400 nM), NEDD8 enzyme 1 inhibitor (NAE1i) $(1 \mu \mathrm{M})$, or JQ1 $(10 \mu \mathrm{M})$ for $2 \mathrm{hrs}$, followed by electroporation of VHL-1/EloB ${ }^{\mathrm{C} 89 S} / \mathrm{EloC}$ and co-incubation for $6 \mathrm{hrs.}$

F. Immunoblot analysis of HEK293A cells electroporated with $\mathrm{VHL}-1 /$ EloB $^{\mathrm{C} 89 S} / \mathrm{EloC}$, with cells collected at $6,24,48$, and 72 hrs post-electroporation.

\section{Figure 3. Degradation of Kinases by Dasatinib-Functionalized VHL}

A. Chemical structure of Compound 2, a maleimide-dasatinib probe.

B. Intact mass spectrometry confirms complete and specific labeling of $\mathrm{VHL}$ by 2. Unconjugated $\mathrm{VHL} / \mathrm{EloB}^{\mathrm{C} 89 \mathrm{~S}} / \mathrm{EloC}$ is shown in blue, and $\mathrm{VHL}-2 / \mathrm{EloB}^{\mathrm{C} 895} / \mathrm{EloC}$ in green.

C. Immunoblot analysis showing electroporation of $\mathrm{VHL}-2 / \mathrm{EloB}^{\mathrm{C} 89 \mathrm{~S}} / \mathrm{EloC}$ into HEK293A cells led to dose-dependent decreases in ABL1 and Lyn, as compared to cells electroporated with buffer, BSA-2, or unlabeled VHL/EloB ${ }^{\mathrm{C} 89 S} /$ EloC. Doses are represented as $\mu \mathrm{g}$ of recombinant protein electroporated per well.

D. Immunoblot analysis of HEK293A cells pretreated with DMSO, carfilzomib (400 nM), NEDD8 enzyme 1 inhibitor (NAE1i) $(1 \mu \mathrm{M})$, or Dasatinib $(1 \mu \mathrm{M})$ for $2 \mathrm{hrs}$, followed by electroporation

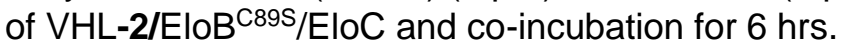

\section{Figure 4. Degradation of Kinases by Dasatinib-Functionalized SPSB2}

A. Intact mass spectrometry demonstrates labeling of SPSB2 by $2(\sim 50 \%)$. Unconjugated SPSB2/EloB/EloC is shown in blue, and SPSB2-2/EloB/EloC in green.

B. SPSB2 Cys53 (highlighted in orange) is solvent exposed and is located near the native substrate binding site. SPSB2 is shown in gray, bound to a reported cyclic peptide inhibitor (cR8), shown in green. Image from PDB 5XN3 (You et al., 2017)

C. Immunoblot analysis showing that electroporation of SPSB2-2/EloB/EloC into HEK293A cells led to a dose-dependent decrease in $A B L$ and Lyn, while electroporation of buffer, BSA-2, or 
SPSB2/EloB/EloC did not affect kinase abundance. Doses are represented as $\mu \mathrm{g}$ of recombinant protein electroporated per well.

D. Immunoblot analysis of HEK293A cells pretreated with DMSO, carfilzomib (400 nM), NEDD8 enzyme 1 inhibitor (NAE1i) $(1 \mu \mathrm{M})$, or Dasatinib $(1 \mu \mathrm{M})$ for $2 \mathrm{hrs}$, followed by electroporation of SPSB2-2/EloB/EloC and co-incubation for 6 hrs.

E. Quantitative mass spectrometry proteomics reveals that electroporated SPSB2-2/EloB/EloC promotes the degradation of dasatinib targets, including EPHB2, EPHB4, ABL1, and ABL2. EloB-2 weakly promotes the degradation of EPHB2 and EPHB4, suggesting the potential of this E3 adaptor protein to promote the targeted degradation of neo-substrates. Identified targets of dasatinib $(\mathrm{Ki}<10 \mathrm{nM})$ are depicted as blue crosses.

\section{Acknowledgements}

The authors would like to thank Lei Xu for kindly providing cell lines, and Seth Carbonneau, Michael Salcius and Jennifer Lipps for advising on protocols. Also, many thanks to Catherine Dubreuil and the Harvard Program in Therapeutic Science for enabling an internship for B.J.P., E.P.H., and Z.J.H.

\section{Author Contributions}

L.M.M. and B.J.P. wrote the manuscript, with edits from the team. B.J.P. performed the biological experiments. D.L.B. and P.L.D. synthesized the maleimide probes. S.G. performed the bioinformatics analysis. D.D. and L.T. generated the proteins. S.M.B. and L.M.M. performed the proteomics experiment. E.P.H., Z.J.H., M.S., and E.R.S. provided intellectual contributions regarding project directions. W.C.F., D.D., L.M.M., and C.R.T. initiated and supervised the project, and provided experimental support. C.R.T. conceived of the idea.

\section{METHODS}

Protein Production. The VHL and SPSB2 complexes were expressed from a pETite-based polycistronic vector. Constructs were codon-optimized for $E$. coli and synthesized by Twist Biosciences.

C41(DE3) cells (Lucigen) were transformed with the expression plasmid using standard techniques. A single colony was used to start an overnight culture in LB + kanamycin media. This culture was used to inoculate $1 \mathrm{~L}$ cultures in Terrific Broth, supplemented with $50 \mathrm{mM}$ sodium phosphate $\mathrm{pH} 7.0$ and $50 \mathrm{ug} / \mathrm{mL}$ kanamycin. These cultures grew in Fernbach flasks at 37 degrees $\mathrm{C}$ while shaking at $225 \mathrm{rpm}$, until the OD600 reached approximately 0.625 , at which point the temperature was reduced to 20 degrees $\mathrm{C}$ and $1 \mathrm{mM}$ IPTG (final) was added to each culture. The cells were allowed to grow overnight.

Cells were harvested by centrifugation at $6,000 \times \mathrm{g}$ for 20 minutes and resuspended in Lysis buffer (25 mM Tris pH 8.0, $400 \mathrm{mM} \mathrm{NaCl}, 1 \mathrm{mM}$ TCEP). Cells were lysed by 3 passes through a cell homogenizer at $18,000 \mathrm{psi}$. Cell lysate was clarified by centrifugation at 160,000 $\mathrm{xg}$ for 2 hours. The whole cell lysate, and then the pellet (following centrifugation) were usually observed to be milky white in appearance, due to the presence of large quantities of inclusion bodies containing the E3 ligase and/or elongins. However, despite the presence of these inclusion bodies (which are removed in this step) there was considerable quantities of soluble protein complex.

Clarified lysate was supplemented with $20 \mathrm{mM}$ imidazole (final) and was then flowed through Ni-NTA resin ( $5 \mathrm{~mL}$ bed volume, gravity fed). The resin was pre-equilibrated with $5 \mathrm{CV}$ of Lysis buffer. After binding of the lysate, the resin was washed with $5 \mathrm{CV}$ of Lysis Buffer, followed by $5 \mathrm{CV}$ of Wash buffer (25 mM Tris pH 8.0, $400 \mathrm{mM} \mathrm{NaCl}, 40 \mathrm{mM}$ imidazole, $1 \mathrm{mM}$ TCEP), followed by elution with 5 CV of Elution buffer (25 mM Tris pH 8.0, $400 \mathrm{mM} \mathrm{NaCl}, 500 \mathrm{mM}$ imidazole, $1 \mathrm{mM}$ TCEP). 
The IMAC eluate was treated with HRV 3C protease. This reaction mixture was placed into a 10,000 MWCO dialysis cassette and dialyzed against Lysis Buffer overnight at $4^{\circ} \mathrm{C}$. Cleavage was confirmed in the morning by ESI-LC/MS.

The protein was then subjected to reverse-IMAC purification by flowing through $3 \mathrm{~mL}$ of Ni-NTA resin pre-equilibrated with Lysis buffer. Upon flow-through, an additional $7 \mathrm{~mL}$ of Lysis Buffer was flowed over the resin to recover the remainder of the protein. The flow-through was then concentrated with a centri-con 3,000 MWCO concentrator (Millipore) to approximately $2 \mathrm{~mL}$.

The protein was lastly subjected to Size Exclusion Chromatography utilizing a Superdex 75 16/60 column, pre-equilibrated with SEC Buffer. Protein was loaded and run at $1 \mathrm{~mL} / \mathrm{min}$. The complex eluted as a single peak, well separated from the void peak. Pure fractions from this peak were combined and concentrated to $10.7 \mathrm{mg} / \mathrm{mL}$ (by nanodrop) and aliquoted

Cell Culture. HEK293A (Invitrogen) and 293FT (Invitrogen, cat \# R70007) were cultured in DMEM supplemented with $10 \%$ fetal bovine serum (FBS) and 1\% penicillin/streptomycin. $786-\mathrm{O}$ cells (ATCC) were cultured in RPMI 1640 supplemented with 10\% FBS and 1\% penicillin/streptomycin. All cell lines were cultured at $37^{\circ} \mathrm{C}$ in a humidified chamber.

Generation of VHL-expressing 786-O cells. Mcherry in a pENTR plasmid (Thermo Scientific, cat \# A10462) and pENTR221-VHL (Thermo Scientific, NM_198156) were Gateway LR cloned into the destination vector pLENTI4/V5 DEST (ThermoFisher Scientific, cat \# V49810). Each plasmid was co-transfected with ViraPower ${ }^{\mathrm{TM}}$ Lentiviral Packaging Mix (ThermoFisher Scientific, cat \# K497500) into 293FT cells to make virus particles. Virus particles were filtered through a 0.4 micron filters and used to infect 786-0 cells. Selection was performed with $500 \mu \mathrm{g} / \mathrm{ml}$ of Zeocin for two weeks to obtain 786-0 VHL and 786-0 mcherry pooled cell lines.

Immunoblotting. Whole cell lysates for immunoblotting were prepared by pelleting cells at $4^{\circ} \mathrm{C}(300$ g) for 3 minutes. Cell pellets were then washed 1x with PBS and resuspended in RIPA lysis buffer (VWR, cat \# 97063-270) supplemented with protease (Thermo Scientific, cat \# A32955) and phosphatase (Thermo Scientific, cat \# A32957) inhibitor tablets. Lysates were clarified at 13,200 rpm for $15 \mathrm{~min}$ at $4^{\circ} \mathrm{C}$ prior to quantification by Lowry assay (Bio-Rad cat \# 5000113 and cat \# 5000114). Whole cell lysates were loaded into 4-20\% Criterion TGX Precast 18 well gels (Bio-Rad, cat \# $5671094)$ and separated by electrophoreses at $120 \mathrm{~V}$ for $1 \mathrm{hr}$. The gels were transferred to a nitrocellulose membrane using the Trans-Blot Turbo (Bio-Rad) for 7 minutes and then blocked for 1 hr at room temperature in Odyssey blocking buffer (LICOR Biosciences, cat \# 927-50000). Membranes were probed with the appropriate primary antibodies (diluted $1: 1000$ ) overnight at $4^{\circ} \mathrm{C}$ in $20 \%$ Odyssey blocking buffer in 1x TBST. Membranes were washed three times with 1x TBST (5 minutes per wash), and then incubated with IRDye goat anti-mouse (LICOR, cat \# 926-32210) or goat anti-rabbit (LICOR, cat \# 926-32211) secondary antibody diluted 1:10,000 in 20\% Odyssey blocking buffer for $1 \mathrm{hr}$ at room temperature. After three 5-minute washes with 1x TBST, the immunoblots were visualized using the ODYSSEY Infrared Imaging System (LICOR). Antibodies used were as follows: Lyn (Cell Signaling, cat \# 2796), VHL (Cell Signaling, cat \# 68547), HIF2 $\alpha$ (Cell Signaling, cat \# 7096), a -Tubulin (Cell Signaling, cat \# 3973), BRD4 (Cell Signaling, cat \# 13440), CUL2 (Invitrogen, cat \# 51-1800), RBX1 (Cell Signaling, cat \# 4397), and ABL1 (Cell Signaling, cat \# 2862).

Electroporation Optimization by FACS analysis. The pre-programmed 24-well Neon Transfection System protocol using the Neon $10 \mu \mathrm{L}$ tips (Invitrogen, cat \# MPK1096) was used to optimize the electroporation conditions for both HEK293A and 786-O cells. Cells were trypsinized and resuspended in media with $10 \%$ FBS but without penicillin/streptomycin prior to counting. Cells were then washed $1 \mathrm{x}$ with $5 \mathrm{~mL}$ PBS before resuspending in Neon resuspension buffer plus Alexa Fluor 
488-labeled BSA, in preparation for $24 \times 10 \mu \mathrm{L}$ electroporation reactions (using the Neon preprogrammed 24 optimization conditions), with 250,000 cells and 4.5 ug of Alexa Fluor 488-labeled BSA electroporated per $10 \mu \mathrm{L}$ reaction. Following electroporation, cells were immediately transferred to a 24-well plate containing $500 \mu \mathrm{L}$ of pre-warmed media. After a $16 \mathrm{~h}$ incubation, cells were washed $1 \mathrm{x}$ with $100 \mu \mathrm{L}$ of PBS prior to trypsinizing ( $80 \mu \mathrm{L}$ of TrpLE Express per well). Trypsinized cells were then neutralized with $150 \mu \mathrm{L}$ of PBS $+20 \%$ FBS, mixed by pipetting, and transferred to a 96-well V-bottom plate (Corning, cat \# 3894) for FACS analysis. Samples were analyzed using a CytoFLEX benchtop flow cytometer using the FITC channel.

Electroporation for Immunoblot Analysis. Cells were trypsinized and resuspended in media with $10 \%$ FBS but without penicillin/streptomycin prior to counting. Cells were then washed $1 \times$ with $5 \mathrm{~mL}$ PBS. Each cell pellet was then resuspended in Neon resuspension buffer plus the indicated concentration of recombinant protein to give a final volume of $130 \mu \mathrm{L}$ per condition (this includes excess volume to avoid bubbles). The cells were then electroporated with the Neon transfection system using the Neon $100 \mu \mathrm{L}$ kit (Invitrogen, cat \# MPK10096) and transferred to a 6-well plate containing $2 \mathrm{~mL}$ of pre-warmed media. 2 million cells were electroporated per reaction. The Neon electroporation parameters used were:

1) 786-O cells: Pulse Voltage: 1150; Pulse Width: 30; Pulse Number: 2.

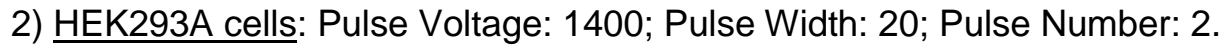

Cells were collected at the indicated time points following electroporation. To collect cells, each well was washed $1 \mathrm{x}$ with $1 \mathrm{~mL}$ PBS, followed by trypsinization with $250 \mu \mathrm{L}$ TrpLE Express (gibco, cat \# 12605010). The trypsin was then neutralized with $250 \mu \mathrm{L}$ media. Cells were washed $1 \mathrm{x}$ with $500 \mu \mathrm{L}$ PBS and then lysed with $50 \mu \mathrm{L}$ RIPA lysis buffer supplemented with protease and phosphatase inhibitors. Samples were normalized and prepped in 4x LDS (Invitrogen, cat \# NP0007) + 10\% NuPage sample reducing agent (Invitrogen, cat \# NP0009) and boiled for $5 \mathrm{~min}$ at $95^{\circ} \mathrm{C}$. Lysates were probed for the specified proteins by western blotting.

Electroporation Rescue Experiments. Cells were plated in $10 \mathrm{~cm}$ plates with 5 million cells per plate in $6 \mathrm{~mL}$ of media. The day after plating, each plate was incubated with the indicated pre-treatment condition (DMSO, carfilzomib, MLN4924, JQ1, or dasatinib) at the indicated concentration for 2 hrs. Cells were trypsinized and resuspended in media, followed by electroporation, as detailed above. Following electroporation, cells were transferred to a 6 -well plate containing $2 \mathrm{~mL}$ of pre-warmed media and were immediately re-treated with the corresponding co-treatment condition, followed by a $6 \mathrm{hr}$ incubation. Cells were then collected as detailed above.

Biotinylation of VHL/EloB ${ }^{\mathrm{C} 89 \mathrm{~S}} / \mathrm{EloC}$ in preparation for Co-Immunoprecipitation. Avi-tagged

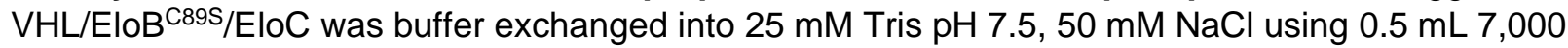
molecular weight cutoff Zeba columns (Thermo Scientific, cat \# PI89882), in preparation for biotinylation. $837 \mathrm{ug}$ of VHL/EloBC89S/EloC was incubated with $20 \mu \mathrm{g}$ of GST-tagged biotin ligase (GST-BirA), $1 \mathrm{mM} \mathrm{ATP,} 5 \mathrm{mM}$ D-biotin, and $1 \mathrm{mM} \mathrm{MgCl} 2$ overnight at $4^{\circ} \mathrm{C}$ (with rotation). The reaction was monitored by intact mass spectrometry. The sample was separated over a 3-minute gradient of $5 \%$ to $60 \%$ acetonitrile $+0.04 \%$ trifluoroacetic acid (TFA) in water $+0.05 \%$ TFA. Mass spectra are acquired over a mass range of $700 \mathrm{~m} / \mathrm{z}$ to $3000 \mathrm{~m} / \mathrm{z}$. The spectra were then deconvoluted over the depicted mass ranges using Maximum Entropy (MaxEnt). Following reaction completion, the biotinylation mixture was then incubated with $100 \mu \mathrm{L}$ of a 1:1 glutathione resin (Cytiva, cat \# 17075601): buffer (25 mM Tris pH 7.5, $50 \mathrm{mM} \mathrm{NaCl}$ ) slurry for $1 \mathrm{~h}$ at room temperature. Following removal of the glutathione beads by centrifuging in a centrifugal spin column (VWR, modified Nylon membrane, $0.45 \mu \mathrm{m}$ ) at $1.5 \mathrm{rcf}$ for $2 \mathrm{~min}$, the protein was buffer exchanged using a $0.5 \mathrm{~mL}$ 7,000 molecular weight cutoff Zeba column into $25 \mathrm{mM}$ HEPES, pH 7.4, $150 \mathrm{mM}$ 
$\mathrm{NaCl}, 0.5 \mathrm{mM}$ EDTA. Protein concentration was determined by Nanodrop using the Protein A280 method.

Co-Immunoprecipitation. 786-O cells were trypsinized and resuspended in RPMI media with 10\% FBS but without penicillin/streptomycin, prior to counting. Cells were then washed $1 \mathrm{x}$ with $5 \mathrm{~mL}$ PBS. Each cell pellet was then resuspended in Neon resuspension buffer plus either buffer $(25 \mathrm{mM}$ HEPES, pH 7.4, $150 \mathrm{mM} \mathrm{NaCl}, 0.5 \mathrm{mM}$ EDTA), biotin-VHL/EloBC89S/EloC (15 $\mu \mathrm{g}$ per $100 \mu \mathrm{L}$ electroporation reaction), or VHL/EloBC89S/EloC (15 $\mu$ g per $100 \mu \mathrm{L}$ electroporation reaction) to give a final volume of $130 \mu \mathrm{L}$ per condition (this includes excess volume to avoid bubbles), with $n=2$ electroporation samples per condition. The cells were then electroporated with the Neon transfection system using the Neon $100 \mu \mathrm{L}$ kit (Invitrogen, cat \# MPK10096), with the following electroporation program: Pulse Voltage: 1150; Pulse Width: 30; Pulse Number: 2. Following electroporation, cells were transferred to a 6 -well plate containing $2 \mathrm{~mL}$ of pre-warmed media and were incubated for 6 hours. 1 million cells were electroporated per $100 \mu \mathrm{L}$ reaction. To collect cells, each well was washed $1 x$ with $1 \mathrm{~mL}$ PBS, followed by trypsinization with $250 \mu \mathrm{L}$ TrpLE Express (gibco, cat \# 12605010). The trypsin was then neutralized with $250 \mu \mathrm{L}$ media, and the $\mathrm{n}=2$ samples per condition were pooled. Cells were washed $1 \mathrm{x}$ with $500 \mu \mathrm{L}$ PBS and then lysed with $100 \mu \mathrm{L}$ Pierce IP lysis buffer (Thermo Scientific, cat \# 87787), supplemented with protease and phosphatase inhibitors. Samples were clarified and normalized to $160 \mu \mathrm{L}$ of $0.65 \mathrm{mg} / \mathrm{mL}$ per condition. $10 \mu \mathrm{L}$ of lysate for each condition was reserved and combined with $10 \mu \mathrm{L}$ of $2 x \mathrm{LDS}+5 \%$ NuPage sample reducing agent, boiled for $5 \mathrm{~min}$ at $95^{\circ} \mathrm{C}$, and loaded as the input control $(2.6 \mu \mathrm{g}$ of total lysate per well of the gel). To the remaining $150 \mu \mathrm{L}$ of lysate, was added $30 \mu \mathrm{L}$ of a $1: 1$ streptavidin agarose resin (Thermo Scientific, cat \# 20353): Pierce IP buffer slurry. The lysates were incubated with the streptavidin agarose resin for $1 \mathrm{~h}$ at $4^{\circ} \mathrm{C}$ (with rotation). Beads were washed $4 \mathrm{x}$ with $500 \mu \mathrm{L}$ of Pierce IP buffer. For the final wash, beads were rotated in the washing buffer for $3 \mathrm{~min}$ at $4^{\circ} \mathrm{C}$. Beads were then pelleted by centrifugation (3,000 rpm for $1 \mathrm{~min}$ ), dried, and boiled for $5 \mathrm{~min}$ at $95^{\circ} \mathrm{C}$ in $30 \mu \mathrm{L}$ of $2 x$ LDS $+5 \%$ NuPage sample reducing agent. Lysates were probed for the specified proteins by western blotting.

Covalent Labeling and Monitoring by Intact Mass Spectrometry. Recombinant purified proteins

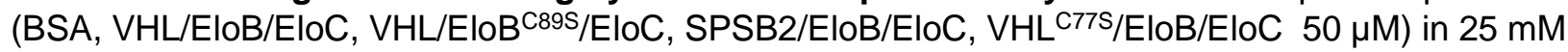
HEPES, pH 7.4, $150 \mathrm{mM} \mathrm{NaCl}, 0.5 \mathrm{mM}$ EDTA were incubated with maleimide probes (Compound 1 or $2 ; 50 \mu \mathrm{M}$ ) at room temperature. BSA was conjugated to Alexa Fluor 488 using Alexa Fluor $488 \mathrm{C}_{5}$ Maleimide (Invitrogen, cat \# A10254). After $2 \mathrm{~h}$, the mass of the resulting complexes was measured by intact protein mass spectrometry, using a Waters Acquity UPLC/ESI-TQD with an Acquity UPLC BEH C4 $2.1 \times 100 \mathrm{~mm}, 1.7$ um column heated to $80 \mathrm{C}$. The sample was separated over a 3-minute gradient of $5 \%$ to $60 \%$ acetonitrile $+0.04 \%$ trifluoroacetic acid (TFA) in water $+0.05 \%$ TFA. Mass spectra are acquired over a mass range of $700 \mathrm{~m} / \mathrm{z}$ to $3000 \mathrm{~m} / \mathrm{z}$. The spectra were then deconvoluted over the depicted mass ranges using Maximum Entropy (MaxEnt). If the reaction was incomplete, more maleimide probe was added until completion. The protein was then passed through a $0.5 \mathrm{~mL} 7,000$ molecular weight cutoff Zeba column (Thermo Scientific, cat \# PI89882), using $25 \mathrm{mM}$ HEPES, pH 7.4, $150 \mathrm{mM} \mathrm{NaCl}, 0.5 \mathrm{mM}$ EDTA as the equilibrating buffer, in order to remove any unreacted maleimide probe. Protein concentration was determined by Nanodrop using the Protein A280 method.

Labeling Site Identification by Peptide Mapping. SPSB2-2/EloB/EloC (50 $\mu \mathrm{L}$ of a $5 \mu \mathrm{M}$ solution) was reduced with $10 \mathrm{mM}$ DTT for 15 minutes at room temperature and alkylated with $25 \mathrm{mM}$ iodoacetamide for 30 minutes at room temperature prior to digestion with $75 \mathrm{ng}$ chymotrypsin at $37 \mathrm{C}$ for $4 \mathrm{~h}$. The sample was acidified with formic acid to $0.1 \% \mathrm{v} / \mathrm{v}$ and was analyzed on a Thermo Orbitrap Q Exactive Mass Spectrometer (Xcalibur 4.1) coupled to an Easy-nLC 1000 high- 
performance liquid chromatography system (Thermo Fisher Scientific). A Kasil-fritted trapping column (75 $\mu \mathrm{m} \times 15 \mathrm{~mm}$ ) packed with 5 um ReproSil-Pur $120 \mathrm{C18}-\mathrm{AQ}$, was used together with a fused silica spraying capillary pulled to a tip diameter of 8-10 um using a P-2000 capillary puller (Sutter Instruments). The capillary tubing (75 $\mu \mathrm{m}$ I.D.) was packed with a $120 \mathrm{~mm}$ separation column comprised of 3 um ReproSil-Pur C18 AQ. Samples $(10 \mu \mathrm{L})$ were injected onto the trapping column using $0.1 \%$ formic acid $/ 2 \%$ acetonitrile in water at a flow rate of $2.5 \mu \mathrm{L} / \mathrm{min}$. Trapped peptides were then introduced into the separation column and eluted at $300 \mathrm{~nL} / \mathrm{min}$ using a mobile phase $\mathrm{A}: 2 \%$ acetonitrile $+0.1 \%$ formic acid in water and a mobile phase $\mathrm{B}: 98 \%$ acetonitrile $+0.1 \%$ formic acid in water with the following gradient (3-7\% B over 30 s followed by $7-45 \%$ B over 36 min 30 s followed by $45-75 \%$ B over 30 s followed by $75 \%$ B for $5 \mathrm{~min}$ ). Data were acquired on Q Exactive Mass Spectrometer in data-dependent mode with MS2 triggered on the top 12 precursor ions in the MS1 scan range of $300-1500 \mathrm{~m} / \mathrm{z}$. Mass spectrometry scans were obtained at 70,000 mass resolution at $200 \mathrm{~m} / \mathrm{z}$ using a target of $1 \mathrm{E} 6$ ions and a maximum fill time of $120 \mathrm{~ms}$. MS2 scans were acquired from 200 to $2000 \mathrm{~m} / \mathrm{z}$ with normalized collision energy of 25 at a resolution of 17,500 at $200 \mathrm{~m} / \mathrm{z}$ with an AGC target of 5E4 ions, a maximum fill time of $120 \mathrm{~ms}$. and an isolation window of $2.0 \mathrm{~m} / \mathrm{z}$. Dynamic exclusion time $15 \mathrm{~s}$, repeat count 1 . Charge states of unassigned, +1 , and $<+5$ were excluded. Nanospray voltage $4.5 \mathrm{keV}$, with capillary temperature $320 \mathrm{C}$, and S-lens RF 50 and sheath gas 10.

Proteomics. Lysate was generated from previously electroporated HEK293A cell pellets, and 100 ug/sample was reduced, alkylated, and digested with trypsin overnight at 37C. Individual samples were labeled with commercially available TMT Reagents (Thermo Fisher) according to the manufacturer's protocol. TMT labeled samples were then combined and fractionated by high-pH reversed phase chromatography as previously described.(Spradlin, 2019). The resulting fractions were dried by speed vacuum and reconstituted as 7 fractions. These fractions were analyzed by nanoLC-MS/MS with the instrument and LC conditions described above. Data were acquired on $Q$ Exactive Mass Spectrometer in data-dependent mode with MS2 triggered on the top 12 precursor ions in the MS1 scan range of $300-1500 \mathrm{~m} / \mathrm{z}$. Mass spectrometry scans were obtained at 35,000 mass resolution at $200 \mathrm{~m} / \mathrm{z}$ using a target of $1 \mathrm{E} 6$ ions and a maximum fill time of $120 \mathrm{~ms}$. MS 2 scans were acquired from 200 to $2000 \mathrm{~m} / \mathrm{z}$ at a resolution of 17,500 at $200 \mathrm{~m} / \mathrm{z}$, with an AGC target of $5 E 4$ ions, a maximum fill time of $120 \mathrm{~ms}$, a normalized collision energy of 30 and an isolation window of $2.0 \mathrm{~m} / \mathrm{z}$ with dynamic exclusion time 10s, and repeat count 1 . Isotopes and charge states of unassigned, +1 and $<+6$ were excluded. Nanospray voltage $4.5 \mathrm{keV}$, with capillary temperature 320C, and S-lens RF 50 and sheath gas 4. 


\section{REFERENCES}

Alex, A., Piano, V., Polley, S., Stuiver, M., Voss, S., Ciossani, G., Overlack, K., Voss, B., Wohlgemuth, S., Petrovic, A., et al. (2019). Electroporated recombinant proteins as tools for in vivo functional complementation, imaging and chemical biology. ELife 8, e48287.

Brien, G.L., Remillard, D., Shi, J., Hemming, M.L., Chabon, J., Wynne, K., Dillon, E.T., Cagney, G., Van Mierlo, G., Baltissen, M.P., et al. (2018). Targeted degradation of BRD9 reverses oncogenic gene expression in synovial sarcoma. ELife 7, e41305.

Bond, M.J., Chu, L., Nalawansha, D.A., Li, K., and Crews, C.M. (2020). Targeted Degradation of Oncogenic KRASG12C by VHL-Recruiting PROTACs. ACS Cent. Sci.

Bondeson, D.P., Mares, A., Smith, I.E.D., Ko, E., Campos, S., Miah, A.H., Mulholland, K.E., Routly, N., Buckley, D.L., Gustafson, J.L., et al. (2015). Catalytic in vivo protein knockdown by smallmolecule PROTACs. Nature Chemical Biology 11, 611-617.

Chen, D., Gehringer, M., and Lorenz, S. (2018). Developing Small-Molecule Inhibitors of HECT-Type Ubiquitin Ligases for Therapeutic Applications: Challenges and Opportunities. ChemBioChem 19, 2123-2135.

Clift, D., So, C., McEwan, W.A., James, L.C., and Schuh, M. (2018). Acute and rapid degradation of endogenous proteins by Trim-Away. Nat Protoc 13, 2149-2175.

Filippakopoulos, P., Qi, J., Picaud, S., Shen, Y., Smith, W.B., Fedorov, O., Morse, E.M., Keates, T., Hickman, T.T., Felletar, I., et al. (2010). Selective inhibition of BET bromodomains. Nature 468, 1067-1073.

Gadd, M.S., Testa, A., Lucas, X., Chan, K.-H., Chen, W., Lamont, D.J., Zengerle, M., and Ciulli, A. (2017). Structural basis of PROTAC cooperative recognition for selective protein degradation. Nature Chemical Biology 13, 514-521.

Gechijian, L.N., Buckley, D.L., Lawlor, M.A., Reyes, J.M., Paulk, J., Ott, C.J., Winter, G.E., Erb, M.A., Scott, T.G., Xu, M., et al. (2018). Functional TRIM24 degrader via conjugation of ineffectual bromodomain and VHL ligands. Nature Chemical Biology 14, 405-412.

Hon, W.C., Wilson, M.I., Harlos, K., Claridge, T.D., Schofield, C.J., Pugh, C.W., Maxwell, P.H., Ratcliffe, P.J., Stuart, D.I., and Jones, E.Y. (2002). Structural basis for the recognition of hydroxyproline in HIF-1 alpha by pVHL. Nature 417.

Iliopoulos, O., Kibel, A., Gray, S., and Kaelin, W.G. (1995). Tumour suppression by the human von Hippel-Lindau gene product. Nature Medicine 1, 822-826.

Iliopoulos, O., Ohh, M., Kaelin, W.G. (1998). pVHL 19 is a biologically active product of the von Hippel-Lindau gene arising from internal translation initiation. PNAS 95, 11661-11666.

Jiang, B., Wang, E.S., Donovan, K.A., Liang, Y., Fischer, E.S., Zhang, T., and Gray, N.S. (2019). Development of Dual and Selective Degraders of Cyclin-Dependent Kinases 4 and 6. Angewandte Chemie International Edition 58, 6321-6326.

Klaeger, S., Heinzlmeir, S., Wilhelm, M., Polzer, H., Vick, B., Koenig, P.-A., Reinecke, M., Ruprecht, B., Petzoldt, S., Meng, C., et al. (2017). The target landscape of clinical kinase drugs. Science 358. 
Kuang, Z., Lewis, R.S., Curtis, J.M., Zhan, Y., Saunders, B.M., Babon, J.J., Kolesnik, T.B., Low, A., Masters, S.L., Willson, T.A., et al. (2010). The SPRY domain-containing SOCS box protein SPSB2 targets iNOS for proteasomal degradation. J Cell Biol 190, 129-141.

Lai, A.C., Momar, T., Doris, H., Jemilat, S., Saul, J.-F., Eunhwa, K., John, H., and M., C.C. (2015). Modular PROTAC Design for the Degradation of Oncogenic BCR-ABL.

Angewandte Chemie International Edition 55, 807-810.

Leonard, J.T., Rowley, J.S.J., Eide, C.A., Traer, E., Hayes-Lattin, B., Loriaux, M., Spurgeon, S.E., Druker, B.J., Tyner, J.W., and Chang, B.H. (2016). Targeting BCL-2 and ABL/LYN in Philadelphia chromosome-positive acute lymphoblastic leukemia. Science Translational Medicine 8, 354ra114354 ra114.

Li, Z., Pinch, B.J., Olson, C.M., Donovan, K.A., Nowak, R.P., Mills, C.E., Scott, D.A., Doctor, Z.M., Eleuteri, N.A., Chung, M., et al. (2020). Development and Characterization of a Wee1 Kinase Degrader. Cell Chemical Biology 27, 57-65.

Liu, J. \& Nussinov, R. (2009). The mechanism of ubiquitination in the Cullin-RING E3 Ligase Machinery: Conformational Control of Substrate Orientation. PLoS Comput Biol. 5(10), e1000527.

McDonald, E.R., de Weck, A., Schlabach, M.R., Billy, E., Mavrakis, K.J., Hoffman, G.R., Belur, D., Castelletti, D., Frias, E., Gampa, K., et al. (2017). Project DRIVE: A Compendium of Cancer Dependencies and Synthetic Lethal Relationships Uncovered by Large-Scale, Deep RNAi Screening. Cell 170, 577-592.

Montenegro, R.C., Howarth, A., Ceroni, A., Fedele, V., Farran, B., Mesquita, F.P., Frejno, M., Berger, B.-T., Heinzlmeir, S., Sailem, H.Z., et al. (2020). Identification of molecular targets for the targeted treatment of gastric cancer using dasatinib. Oncotarget 11, 535-549.

Nowak, R.P., DeAngelo, S.L., Buckley, D., He, Z., Donovan, K.A., An, J., Safaee, N., Jedrychowski, M.P., Ponthier, C.M., Ishoey, M., et al. (2018). Plasticity in binding confers selectivity in ligandinduced protein degradation. Nature Chemical Biology 14, 706-714.

Ottis, P., Toure, M., Cromm, P.M., Ko, E., Gustafson, J.L., and Crews, C.M. (2017). Assessing Different E3 Ligases for Small Molecule Induced Protein Ubiquitination and Degradation. ACS Chem. Biol. 12, 2570-2578.

Pozzebon, M.E., Varadaraj, A., Mattoscio, D., Jaffray, E.G., Miccolo, C., Galimberti, V., Tommasino, M., Hay, R.T., and Chiocca, S. (2013). BC-box protein domain-related mechanism for VHL protein degradation. PNAS 110, 18168-18173.

So, R.W.L., Chung, S.W., Lau, H.H.C., Watts, J.J., Gaudette, E., Al-Azzawi, Z.A.M., Bishay, J., Lin, L.T.-W., Joung, J., Wang, X., et al. (2019). Application of CRISPR genetic screens to investigate neurological diseases. Molecular Neurodegeneration 14, 41.

Remillard, D., Buckley, D.L., Paulk, J., Brien, G.L., Sonnett, M., Seo, H.S., Dastjerdi, S., Wühr, M., Dhe-Paganon, S., Armstrong, S.A., et al. (2017). Degradation of the BAF Complex Factor BRD9 by Heterobifunctional Ligands. Angewandte Chemie - International Edition 56, 5738-5743.

Schapira, M., Calabrese, M.F., Bullock, A.N., and Crews, C.M. (2019). Targeted protein degradation: expanding the toolbox. Nature Reviews Drug Discovery 18, 949-963. 
Schönenberger, C., Schütz, A., Franco-Obregón, A., Zenobi-Wong, M. (2011). Efficient electroporation of peptides into adherent cells: investigation of the role of mechano-growth factor in chondrocyte culture. Biotechnol Lett 33(5), 883-888.

Słabicki, M., Kozicka, Z., Petzold, G., Li, Y.-D., Manojkumar, M., Bunker, R.D., Donovan, K.A., Sievers, Q.L., Koeppel, J., Suchyta, D., et al. (2020). The CDK inhibitor CR8 acts as a molecular glue degrader that depletes cyclin K. Nature 1-5.

Spradlin, J.N., Hu, X., Ward, C.C., Brittain, S.M., Jones, M.D., Ou, L., To, M., Proudfoot, A., Ornelas, E., Woldegiorgis, M., et al. (2019). Harnessing the anti-cancer natural product nimbolide for targeted protein degradation. Nature Chemical Biology 15, 747-755.

Tarade, D., Lee, J.E., and Ohh, M. (2019). Evolution of metazoan oxygen-sensing involved a conserved divergence of VHL affinity for HIF1 $\alpha$ and HIF2 $\alpha$. Nature Communications 10, 3293.

Tong, B., Spradlin, J.N., Novaes, L.F.T., Zhang, E., Hu, X., Moeller, M., Brittain, S.M., McGregor, L.M., McKenna, J.M., Tallarico, J.A., et al. (2020). A Nimbolide-Based Kinase Degrader Preferentially Degrades Oncogenic BCR-ABL. ACS Chem. Biol.

Tsong, T.Y. (1989). Electroporation of Cell Membranes. In Electroporation and Electrofusion in Cell Biology, E. Neumann, A.E. Sowers, and C.A. Jordan, eds. (Boston, MA: Springer US), pp. 149-163.

Ward, C.C., Kleinman, J.I., Brittain, S.M., Lee, P.S., Chung, C.Y.S., Kim, K., Petri, Y., Thomas, J.R., Tallarico, J.A., McKenna, J.M., et al. (2019). Covalent Ligand Screening Uncovers a RNF4 E3 Ligase Recruiter for Targeted Protein Degradation Applications. ACS Chem. Biol. 14, 2430-2440.

You, T., Wang, Y., Li, K., Zhang, D., Wei, H., Luo, Y., Li, H., Lu, Y., Su, X., and Kuang, Z. (2017). Crystal structure of SPSB2 in complex with a rational designed RGD-containing cyclic peptide inhibitor of SPSB2-iNOS interaction. Biochemical and Biophysical Research Communications 489, 346-352.

Zecha, J., Meng, C., Zolg, D.P., Samaras, P., Wilhelm, M., and Kuster, B. (2018). Peptide Level Turnover Measurements Enable the Study of Proteoform Dynamics. Molecular \& Cellular Proteomics 17, 974-992.

Zengerle, M., Chan, K.-H., and Ciulli, A. (2015). Selective Small Molecule Induced Degradation of the BET Bromodomain Protein BRD4. ACS Chem. Biol. 10, 1770-1777.

Zhang, X., Crowley, V.M., Wucherpfennig, T.G., Dix, M.M., and Cravatt, B.F. (2019). Electrophilic PROTACs that degrade nuclear proteins by engaging DCAF16. Nature Chemical Biology 15, 737746. 